\title{
CONF-941144--116
}

\section{POROUS SPHERICAL SHELLS AND MICROSPHERES \\ BY ELECTRODISPERSION PRECIPITATION}

\author{
Michael T. Harris, Warren G. Sisson \\ and Osman A. Basaran \\ Chemical Technology Division \\ Oak Ridge National Laboratory* \\ Oak Ridge, Tennessee 37831-6224 \\ Susan M. Hayes and Sophie J. Bobrowski \\ Analytical Services Organization \\ Oak Ridge National Laboratory \\ Oak Ridge, Tennessee 37831-6224
}

Submitted for publication in the Materials Research Society Symposium Proceedings,

Symposium W2- Spheres and Microspheres: Synthesis and Applications

Presented at the 1994 Fall Materials Research Society Meeting

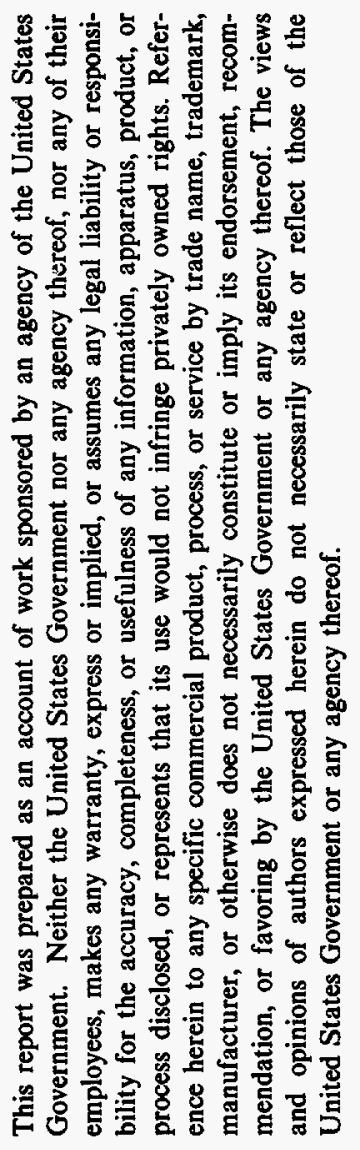

Boston, MA

November 28-December 2, 1994

The submitted manuscript has been authored

by a contractor of the U.S. Government

under contract No. DE-AC05-84OR21400.

Accordingly, the U.S. Government retains a

nonexclusive, royalty-free license to

publish or reproduce the published form

of this contribution, or allow others to

do so, for U.S. Government purposes.

* Managed by Martin Marietta Energy Systems, Inc., under contract DE-AC05-84OR21400 with the U.S. Department of Energy. 


\section{DISCLAIMER}

Portions of this document may be illegible in electronic image products. Images are produced from the best available original document. 


\section{POROUS SPHERICAL SHELLS AND MICROSPHERES BY ELECTRODISPERSION PRECIPITATION}

MICHAEL T. HARRIS, WARREN G. SISSON, SUSAN M. HAYES, SOPHIE J. BOBROWSKI, and OSMAN A. BASARAN

Oak Ridge National Laboratory, Oak Ridge, TN 37831-6224

\section{ABSTRACT}

Pulsed electric fields have been used to enhance the dispersion of aqueous metal ( $Z \mathbf{r}$ and $\mathrm{Al}$ ) salt solutions from a nozzle and into a nonconducting liquid continuous phase that is immiscible with the aqueous phase. The diameter of the resulting microdroplets ranged in size from approximately 0.1 to $10 \mu \mathrm{m}$. Precipitation of hydrous metal oxides occurred as ammonia, which was dissolved in varying amounts in the continuous phase, diffused into the aqueous microdroplets. Spherical shells were formed at higher ammonia concentrations and microspheres were produced at lower ammonia concentrations. Upon drying, dimples appeared in the particles that were synthesized at higher ammonia concentrations. The latter result accords with the well known fact that under certain conditions spherical shells collapse when a fluid is extracted from the core of the particle. No dimples were observed in the microspheres that were produced at lower ammonia concentrations. Analog X-ray dot maps for aluminum and zirconium were done to determine the spatial distribution of each metal in the particles.

\section{INTRODUCTION}

The ability to reproduce the synthesis of dense- and porous-microspheres ( $<1 \mathrm{~mm}$ in diameter) and micron-sized spherical shells is very important in (a) the development of ceramics for structural(1), electronic $(2,3)$, catalyst $(2,4)$ and thermal applications $(5,6)$; and (b) the encapsulation of products for controlled-release of drugs $(7,8,9)$, flavors and perfumes $(2,3,9)$, and inks and dyes $(10)$, and the protection of light-sensitive components and mechanical support of fragile materials (2). Larger ( $>1 \mathrm{~mm}$ in diameter) metallic- and ceramic-spherical shells have been used in inertial confinement fusion (ICF) experiments $(11,12,13)$ and as catalyst supports (4). The current paper will focus on a recent technique that has been developed for synthesizing ceramic microspheres and micro-shells.

The synthesis of ultrafine ceramic precursor powders by physicochemical techniques has gained increasing attention in recent years. In contrast to the strictly chemical method of homogeneous precipitation, these methods [which include spray pyrolysis/aerosol synthesis $(14,15)$, electrostatic atomization of liquid drops into air (16), and precipitation in microemulsions (17)] offer the advantage of physically controlling the particle size by creating localized reaction zones (i.e., microreactors) in which precipitation/gelation occurs. These methods are especially attractive in the synthesis of mixed hydrous metal-oxide precursor powders when chemical homogeneity is required on the submicron level. Methods such as spray pyrolysis/aerosol synthesis (15), electric field-enhanced atomization of liquids in air (16) or liquids (17-22), and precipitation/emulsion-extraction in microemulsions (3) are also viable techniques for synthesizing spherical ceramic shells and microspheres. One process, electrodispersion precipitation which has been pioneered in our research group uses the technique of electric field-enhanced atomization of conductive liquids into a nonconducting liquid to form porous ceramic shells and spheres (19-22). 
Ultrafine hydrous oxide particles of alumina, zirconia, and 1:2:3 yttrium-bariumcopper have been produced in the Electric Dispersion Reactor (EDR) through multiphase electrodispersion precipitation of aqueous salt solutions of those metals into an organic phase containing ammonia (19-22). In this system, a pulsed $\left(5000 \mathrm{~s}^{-1}\right) \mathrm{dc}$ electric field is used to create submicron- to micron-sized droplets of a conducting aqueous metal salt solution emanating from a nozzle into a nonconducting organic-continuous phase. The aqueous droplets serve as microreactors where metal hydroxide precipitation occurs.

A previous study has shown the effect of the electric field strength and ammonia concentration on the size distribution and shape of zirconia particles that were synthesized by electrodispersion precipitation (22). This paper explores the effect of ammonia concentration on the shape of zirconia and mixed zirconia/alumina particles. Such particles have applications in areas as diverse as high temperature catalysis and ion exchange.

\section{EXPERIMENTAL}

A schematic of the process based on the electrodispersion precipitation reactor (EDR) is shown in Figure 1. The major components consist of the organic and aqueous feed containers, the electric dispersion reactor with the associated electrical components, and a collection vessel. The aqueous and organic flow rates were 1 and $100 \mathrm{~mL} / \mathrm{min}$, respectively. A typical peak electrical field strength ranged from 25 to $30 \mathrm{kV} / \mathrm{cm}$ which decayed exponentially upon application of each pulse. The pulsing frequency was 5000 $\mathrm{s}^{-1}$. The total concentration of metal salts in the aqueous feed solution was $1 \mathrm{M}$, and the organic (2-ethyl-1-hexanol) phase contained 0.012 and $0.12 \mathrm{M}$ ammonia. Before drying and aquiring SEM and TEM images, the samples were centrifuged and washed several times to remove the excess organic solvent and by-products.

SEM analog dot mapping of aluminum and zirconium in the particles was done with an HNU Systems, Inc. energy dispersive X-ray spectrometer (Model 5000) that was interfaced with a JEOL JSM-5300 scanning electron microscope. The analog dot map is a 2-dimensional visual representation of the spatial distribution of an element across the surface of a sample.

\section{RESULTS AND DISCUSSIONS}

Figures 2-5 show the effect of the ammonia concentration in the 2-ethyl-1-hexanol continuous phase on the formation of microspheres and micro-shells of zirconia and mixed zirconia-alumina. Microspheres are formed when the concentration of ammonia was $0.012 \mathrm{M}$. This is shown in the scanning electron micrograph (SEM) of Figure 2 and transmission electron micrograph (TEM) of Figure 3 for zirconia and mixed aluminazirconia (60 wt\% alumina) particles, respectively. Figures 4 and 5 show SEM and TEM micrographs of the spherical micro-shells of zirconia and alumina-zirconia, respectively, that are formed when the concentration of ammonia is an order of magnitude higher $(0.12 \mathrm{M})$ in the continuous phase.

The formation of shells by this electrodispersion precipitation process is closely related to the formation of coated- and porous shell-aerosol particles by simultaneous diffusion and chemical reaction(22-25). It is well known in aerosol processes that the reaction between a reactive gas and an aeorsol droplet leads to the formation of spherical shells when the reaction rate is fast compared with diffusion within the droplet and when the solubility of the reaction product is low in the solution. Likewise, the synthesis of microspheres and micro-shells by electrodispersion precipitation involves several steps: (1) the creation of aqueous droplets that are $<10 \mu \mathrm{m}$ in the continuous phase, (2) the diffusion of ammonia from the continuous phase to the droplet, (3a) the diffusion 


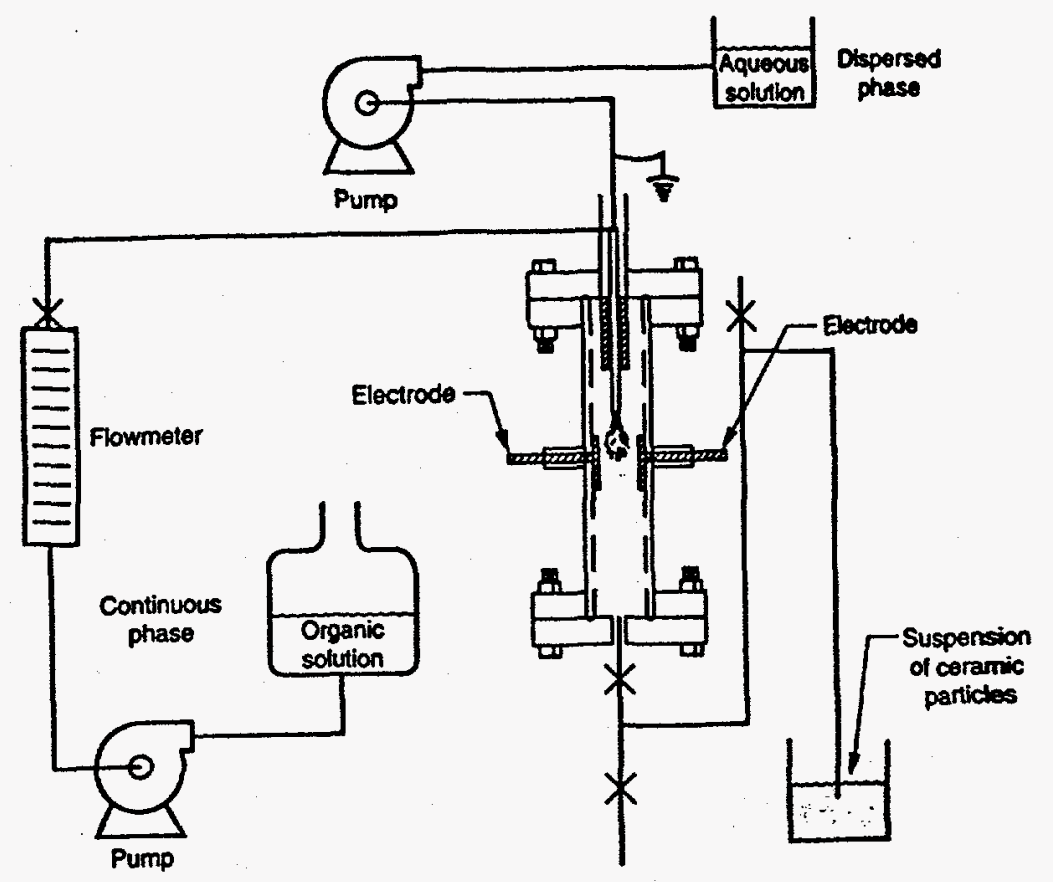

Figure 1. Schematic of EDR process.

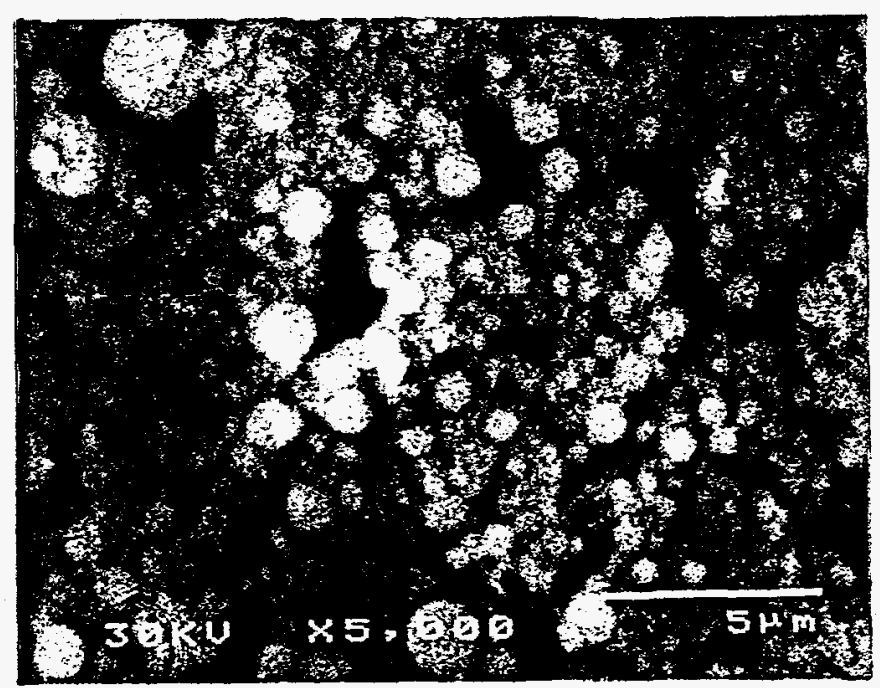

Figure 2. Scanning electron micrograph of zirconia microspheres produced in the EDR by precipitation in an aqueous microdroplet containing $1 \mathrm{M}$ zirconyl nitrate and a continuous phase that contains $0.012 \underline{\mathrm{M}} \mathrm{NH}_{3}$.

of ammonia through the shell and/or (3b) diffusion of ammonia in the droplet and (4) reaction of the ammonia with the metal salt to produce hydroxides. When the ammonia concentration is high, the precipitation reaction is very fast and a shell (Figures 4 and 5 ) is formed which slows the diffusion of ammonia into the core of the sphere. The higher concentration of metal ions in the unreacted core and the lower concentration of metal ions in the shell region causes the metal ions to diffuse to the shell region. Thus, micro-shells are formed at high ammonia concentration where the process is diffusion controlled. At the lower ammonia concentration $(0.012 \underline{\mathrm{M}})$, microspheres (Figures 2 


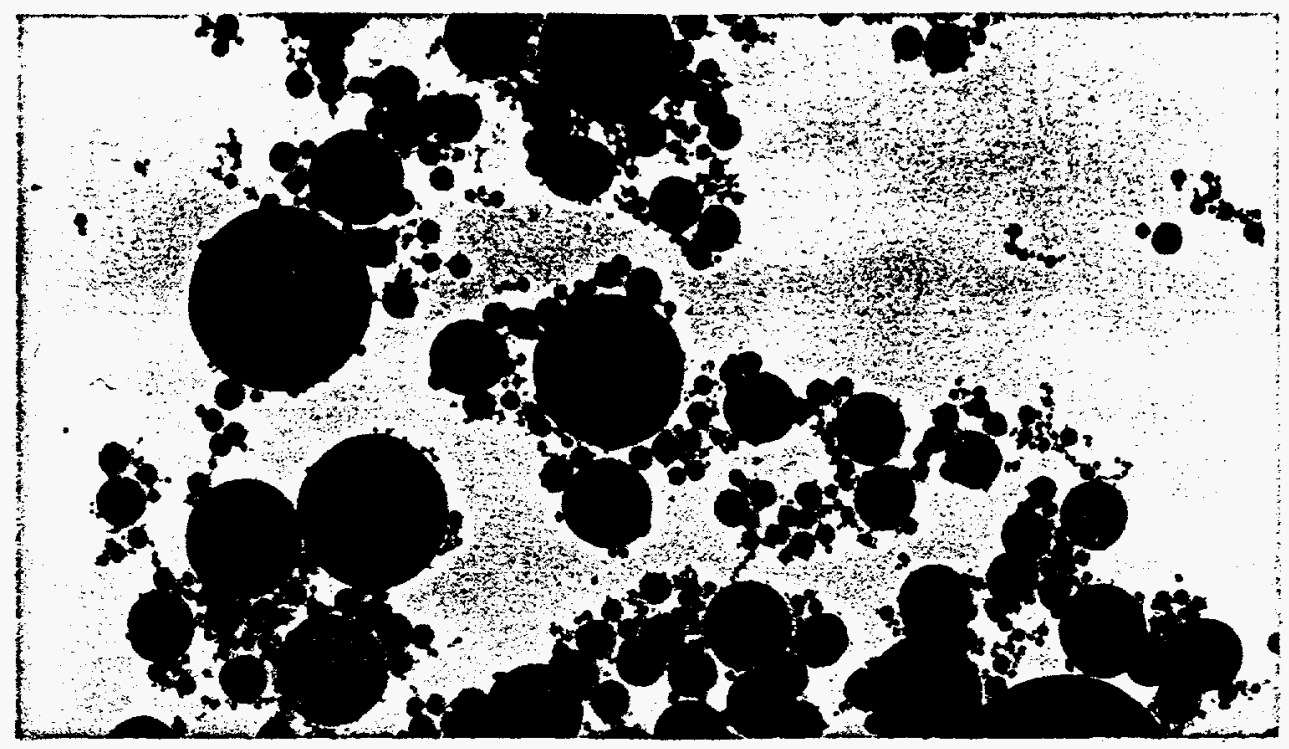

Figure 3. Transmission electron micrograph of alumina:zirconia microspheres produced in the EDR by precipitation in an aqueous microdroplet containing $1 \mathrm{M}$ total metal and a continuous phase that contains $0.012 \underline{\mathrm{M} \mathrm{NH}}$.

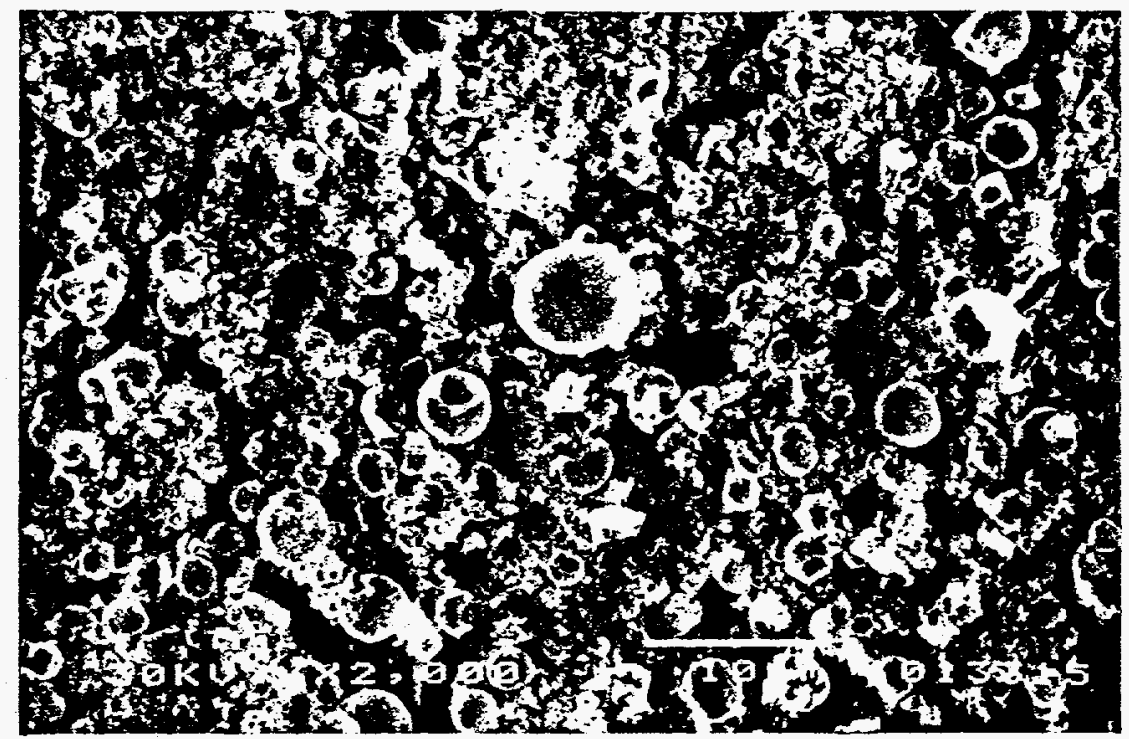

Figure 4. Scanning electron micrograph of zirconia micro-shells produced in the EDR by precipitation in an aqueous microdroplet containing $1 \mathrm{M}$ zirconyl nitrate and a continuous phase that contains $0.12 \underline{\mathrm{M}} \mathrm{NH}_{3}$.

and 3) are formed since the reaction is slow enough for the ammonia to diffuse and precipitation to occur throughout the droplet.

An EDX-aluminum and zirconium analog dot mapping was done to determine the spatial distribution of zirconium and aluminum in the mixed particles. A typical dot mapping of an alumina:zirconia particle is shown in Figure 6. The data clearly show a uniform distribution of zirconium and aluminum atoms on the surface of the particle. This finding was true for alumina:zirconia microspheres and micro-shells. 


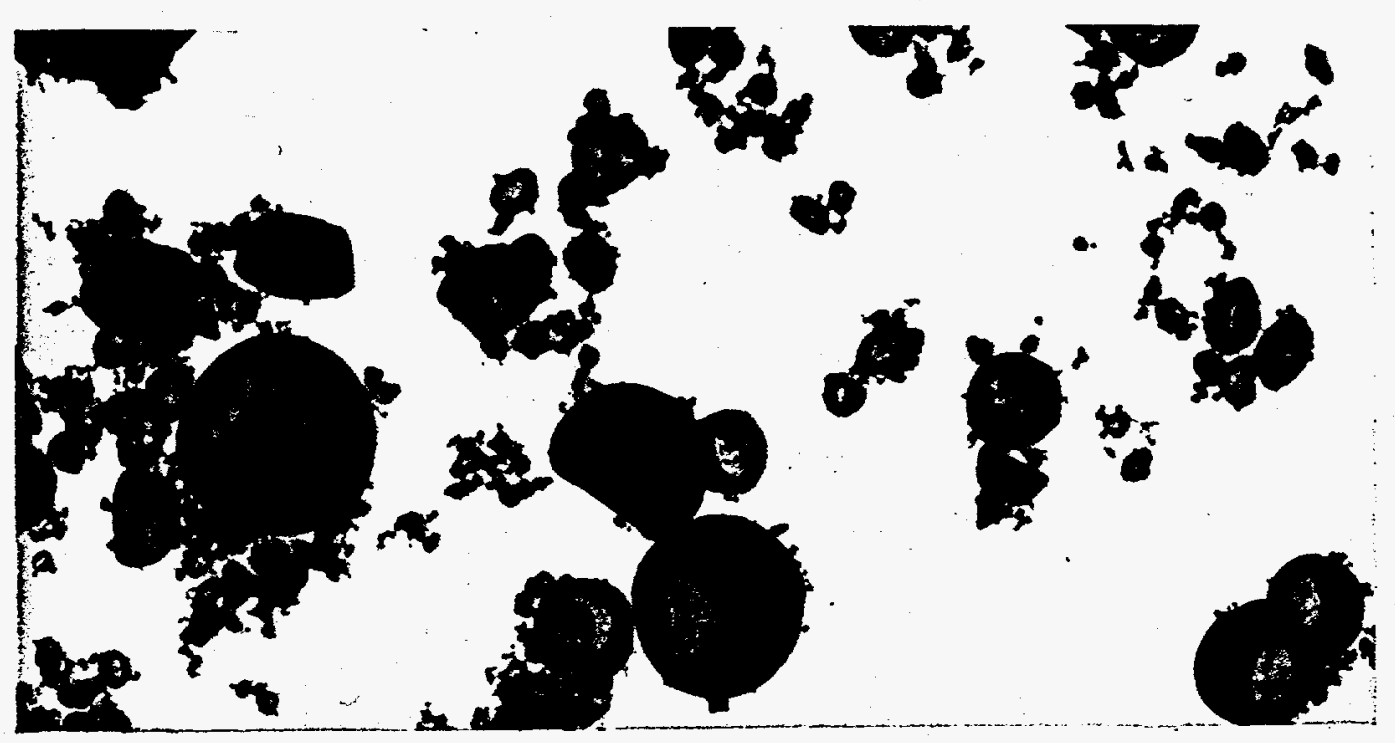

Figure 5. Transmission electron micrograph of alumina:zirconia micro-shells produced in the EDR by precipitation in an aqueous microdroplet containing $1 \underline{\mathrm{M}}$ total metal and a continuous phase that contains $0.12 \underline{\mathrm{M}} \mathrm{NH}_{3}$.
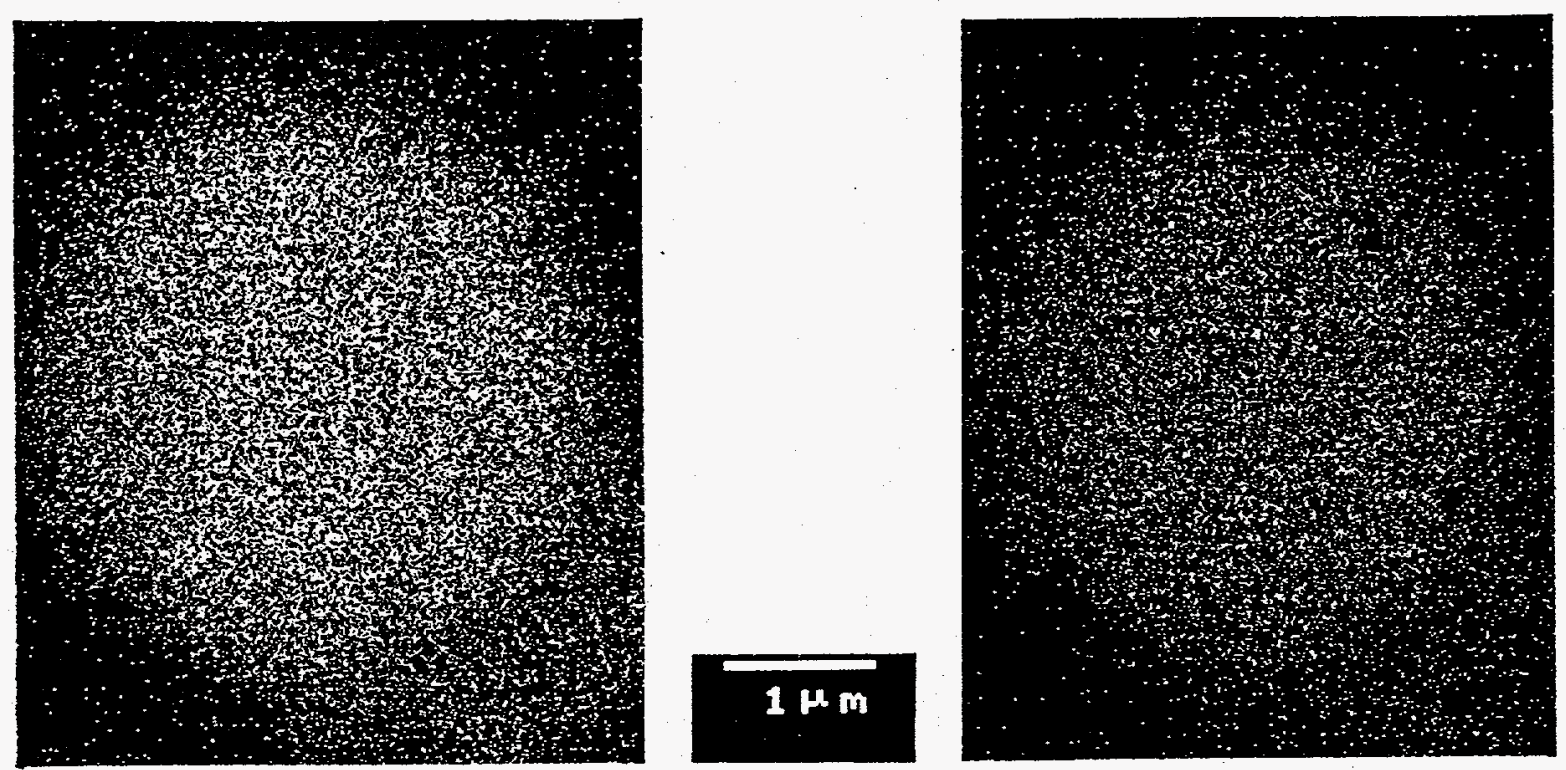

Figure 6. Dot mapping of alumina:zirconia ( $60 \mathrm{wt} \%$ alumina) particles.

\section{CONCLUSIONS}

Microspheres and micro-shells of zirconia and alumina:zirconia have been synthesized by electrodispersion precipitation. The micro-shells are produced when the concentration in the continuous phase is high and microspheres are produced at low ammonia concentration. Additional tests are underway to determine the effect of the ammonia concentration on the thickness of the wall of the micro-shell that is formed during electrodispersion precipitation. 


\section{ACKNOWLEDGMENTS}

This research was sponsored by the Office of Basic Energy Sciences, Division of Materials Sciences and Division of Advanced Energy Project, U.S. Department of Energy under contract DE-AC05-84OR21400 with Martin Marietta Energy Systems, Inc.

\section{REFERENCES}

[1] W.H. Rhodes, J. Am. Ceram. Soc., 64, 19 (1981).

[2] R.L. Downs, M.A. Ebner and W.J. Miller, in Sol-Gel Technology for Thin Films, Fibers, Preforms, Electronics, and Specialty Shapes, edited by L.C. Klein (Noyes Publication, Park Ridge, NJ, 1988), pp. 330-381.

[3] G. Liu and D.L. Wilcox, Sr., Mat. Res. Soc. Symp. Proc., 346, 201 (1994).

4] P. Brown, Am. Ceram. Bull., 72, 98 (1993).

5] D.J. Green, J. Am. Ceram. Soc., 68, 403 (1985).

6] D.J. Green and R.G. Hoagland, J. Am. Ceram. Soc., 68, 395 (1985).

[7] P.I. Lee and W.R. Good, Controlled-Release Technology: Pharmaceutical Applications, (ACS Symposium Series 348, New York, New York, 1987).

[8] M.A. El-Nokaly, D.M. Piatt and B.A. Charpentier, Polymer Delivery Systems: Properties and Applications, (ACS Symposium Series 520, San Francisco, California, 1993).

[9] C.A. Finch, Chem. Ind., 7, 208 (1989).

[10] J. Krieger, Chem. Eng. News, 66, 23 (1988).

[11] K. Kim and K.Y. Jang, J. Am. Ceram. Soc., 74, 1987 (1991).

[12] J.M. Kendall, Proceedings of the Second International Colloquium on Drops and Bubbles, edited by D.H. Le Croissette (JPL Publication 82-7, 1982), p. 79.

[13] C.D. Hendricks, Proceedings of the Second International Colloquium on Drops and Bubbles, edited by D.H. Le Croissette (JPL Publication 82-7, 1982), p. 88.

[14] B.J. Ingebrethsen, E. Matijevic and R.E. Partch, J. Colloid Interface Sci., [95], 228 (1981).

[15] E.B. Slamovich and F.F. Lange, Mat. Res. Soc. Symp. Proc., 121, 257 (1988).

16] W.T. Minehan and G.L. Messing, J. Non-Cryst. Solids, 121, 375 (1990).

17] M.T. Harris, T.C. Scott, and C.H. Byers, U. S. Patent No. 5122360 (16 June 1992).

18 M.T. Harris, T.C. Scott, and C.H. Byers, U. S. Patent No. 5207973 (4 May 1993).

[19] M.T. Harris, T.C. Scott, O.A. Basaran, and C.H. Byers, Mat. Res. Soc. Proc. 121, 257 (1988).

[20] M.T. Harris, W.G. Sisson, and O.A. Basaran, Mat. Res. Soc. Proc. 271, 945 (1992).

21] M.T. Harris, T.C. Scott, and C.H. Byers, Mat. Sci. Eng. A168, 125 (1993).

[22] M.T. Harris, W.G. Sisson, T.C. Scott, O.A. Basaran. C.H. Byers, W. Ren and T.T. Meek, Mat. Res. Soc. Proc., 346, 171 (1994).

[23] W.R. Foss and E.J. Davis, J. Aerosol Sci., 25, 33 (1994).

[24] W. Li, S.D. Rassat, W.R. Foss and E.J. Davis, J. Colloid Interface Sci., 162, 267 (1994).

[25] G.O. Rubel, J. Appl. Phys., 61, 1633 (1987). 